\title{
Professeur Pierre Royer (1917-1995)
}

\author{
Raphaël Rappaport
}

Pierre Royer décédé le 20 janvier 1995 à l'âge de soixante-dix-huit ans, a été pour la pédiatrie française et internationale un maître au sens le plus complet du terme. Il fut pour plusieurs générations un modèle de médecin et de chercheur, deux qualités qu'il a su allier avec exigence, persévérance et lucidité. Il avait en effet compris, dès les années 1950, qu'un nouveau style de pédiatrie devait s'appuyer sur une recherche biomédicale solide. La tradition clinique française devenait à elle seule incapable de renouveler la réflexion au lit du malade sans une approche physiopathologique des maladies et, puisqu'il s'agissait de la pédiatrie, on devrait ajouter sans une référence permanente au développement biologique mais aussi psychoaffectif de l'enfant. La carrière médicale de Pierre Royer s'est inscrite dans l'évolution contemporaine majeure de la médecine française: mise en place du plein temps hospitalier qui alignait enfin notre pratique hospitalo-universitaire sur celle des pays anglo-saxons et création de la recherche biomédicale dans le cadre de l'Institut national de la santé, devenu ensuite l'Inserm. Cette carrière allait se développer autour de l'hôpital des Enfants-Malades, comme une suite à l'action de son maître le Professeur Robert Debré. Elle s'est déroulée parallèlement aux profonds changements de notre médecine, conduits aussi par d'autres grandes personnalités telles que Jean Hamburger, Jean Bernard, Jean Dausset et René Fauvert. Ces hommes ont su entraîner dans leur sillage une nouvelle génération de médecins avides de comprendre, préparés à la recherche et soucieux d'une éthique qui puisse fonder leur action dans les $\mathrm{m} / \mathrm{s} n^{\circ} 5$, vol. 11 , mai 95 nouvelles institutions qui allaient se développer à partir des années 1950 et donner à la médecine française de nouvelles lettres de noblesse.

Le premier ensemble de travaux cliniques qui marquèrent l'époque pionnière, comme se plaisait à le faire remarquer Pierre Royer, a concerné l'étude des troubles de la soif, de l'alcalose avec hypokaliémie, le traitement du diabète sucré en régime libre, le début de l'étude des insuffisances tubulaires rénales, la physiologie de la filtration glomérulaire du nouveau-né prématuré et à terme. Certains de ces travaux furent le point de départ de plusieurs livres qui ont longtemps fait autorité: Ies insuffisances congénitales du tubule. rénal avec A. Prader, Les maladies héréditaires du métabolisme avec M. Lamy et J. Frézal, Les diarrhées du noumisson avec N. Masse. Parallèlement, il organisait un séminaire sur le métabolisme du calcium qui eut une importance décisive sur l'orientation de certaines recherches.

De l'avis de tous, il a été le père fondateur de la néphrologie infantile. La compétence et l'originalité de son école ont rapidement conquis l'approbation des pédiatres étrangers, en particulier américains. Certains travaux ont longtemps fait autorité : description des lésions du syndrome hémolytique et urémique, syndromes néphrotiques acquis et congénitaux, insuffisances tubulaires, ostéodystrophie rénale, oligoméganéphronie. En 1973, il publiait avec ses plus proches collaborateurs le premier Précis de néphrologie pédiatrique. Dans la suite logique de cette recherche clinique foisonnante, il mettait en place la première unité européenne d'hémodialyse et transplantation rénale chez l'enfant.

L'endocrinologie pédiatrique était naissante dans les années 1960. Parmi les grands noms de cette discipline, à côté de A. Prader en Suisse et L.Wilkins à Baltimore, Pierre Royer apportait sa contribution à nouveau originale: hypoaldostéronisme héréditaire, hypercalciurie idiopathique avec nanisme, insuffisance en hormone de croissance, troubles métaboliques liés à l'insuffisance thyroïdienne furent tour à tour étudiés. Son intérêt pour les troubles du métabolisme du calcium aboutit à la rédaction, avec P. Fourman d'un ouvrage longtemps consulté Calcium metabolism and the bone. Ainsi, son attention se portait sur le développement du squelette à la fois sous l'angle de la biologie de la croissance et comme organe profondément impliqué dans l'homéostasie calcique.

Devenu chef de service en 1967, il organisa son service en un vaste ensemble multidisciplinaire, confiant à ses élèves la pleine responsabilité de plusieurs secteurs. Ainsi préparait-il l'évolution de la pédiatrie hospitalière vers une surspécialisation nécessaire au progrès de la réflexion clinique et à la mise en place d'une recherche structurée et compétitive au plan international. A l'évidence, la base commune à cette action était la référence au développement depuis la vie intra-utérine jusqu'à l'âge adulte. La pédiatrie, grâce à Pierre Royer, avait trouvé le fil directeur qui ouvrait les portes passionnantes de la recherche. Celle-ci, d'abord clinique, devint rapidement plus biologique. Même aujourd'hui, alors que règnent les approches moléculaires et cellulaires, cette période encore souvent descrip- 
tive mais déjà fortement imprégnée de réflexion physiopathologique, reste une référence nécessaire à la compréhension des grandes entités morbides et des processus physiologiques fondamentaux. Tout naturellement, il fut nommé directeur d'une unité Inserm, où il organisa, de façon coordonnée, les études du rein en croissance, de la physiologie rénale, de la biologie de la croissance et des récepteurs des hormones polypeptidiques, du métabolisme et de la pharmacologie de la vitamine $\mathrm{D}$. Pendant une dizaine d'années, les plus fécondes et les plus enthousiasmantes, de nombreux médecins et chercheurs passèrent dans cette école et furent ensuite responsables de nouvelles unités ou services en France et à l'étranger. Cet héritage a conduit la pédiatrie et la biologie du développement au meilleur niveau. Toujours exigeant et critique, il pouvait en tirer une légitime fierté.

Il avait compris que cette émergence d'une médecine toujours plus technique et complexe, mais aussi plus performante, devait être accompagnée d'un effort nécessaire de compréhension de l'enfant malade et de son environnement. Cette démarche dont il voulut qu'elle fùt d'abord une recherche fut confiée à Ginette Raimbault et ses collaborateurs. L'approche psychanalytique de la relation médecin-malade supposait confiance et adhésion de la part de l'équipe médicale et du personnel soignant. Pierre Royer sut donner cette impulsion afin de faire connaître les problèmes psychologiques autour de l'enfant atteint de maladies chroniques et aborder la thématique douloureuse mais nécessaire de «l'enfant et la mort». Ce fut la grandeur de ce médecin d'avoir su combiner et faire cohabiter dans un même lieu la biologie et les sciences humaines au bénéfice de l'enfant malade.

Visionnaire, il a véritablement lutté pour que naisse une recherche en pédiatrie. Généreux et ambitieux, il ne pouvait pas échapper à l'attraction des grandes responsabilités nationales et internationales. Présent là où il le jugeait nécessaire, il a voulu rester à l'écart des honneurs excessifs et des charges administratives qui ris- quaient de le couper du terrain, l'hôpital des Enfants-Malades, où il mettait en place, précurseur dans notre pays, un Département médical de pédiatrie. Il a souvent considéré que les heures passées au Comité consultatif de la recherche scientifique et technique (1965-1970) avait été les plus belles de sa vie professionnelle. Il a su y défendre la position des sciences biologiques et médicales. Il fut ensuite conseiller auprès de la DGRST. Parallèlement, il fut un président du Conseil scientifique de l'Inserm respecté et écouté. La force de sa personnalité, sa capacité exceptionnelle à appréhender l'avenir du monde biomédical l'amenèrent à prendre la présidence du Conseil d'administration de l'Institut Pasteur (1973-1981). Aux côtés de Jacques Monod et François Jacob, il joua un rôle essentiel dans le redressement de cette institution. Avec François Gros et ces derniers, il eut à rédiger en 1979 le rapport sur "Sciences de la vie et société" qui attira l'attention du grand public sur les progrès de la biologie, l'essor des biotechnologies et les problèmes de société ainsi posés.

L'Université ne le laissait pas indifférent et il accepta en 1976 la présidence du Comité interministériel pour les questions hospitalo-universitaires et garda longtemps une grande vigilance devant l'évolution parfois chaotique de notre université.

Infatigable et omniprésent, il mit en place l'Association France Hypophyse, organisa avec G. David le CECOS qui rendit possible le développement de l'insémination artificielle en France, et élabora avec A. Boué les structures nécessaires au diagnostic anténatal précoce.

En 1983, il prit la direction du Centre international de l'enfance, créé par Robert Debré et devenu l'instrument de notre action pour l'enfance défavorisée, en particulier dans les régions les plus démunies du monde. Cela devait aboutir à un engagement vigoureux dans les problèmes d'éthique soulevés par les progrès de la médecine des enfants, dont témoigne un livre resté indispensable en France, Éthique et pédiatrie. Il devait aussi être appelé à siéger au Comité national d'éthique. Il avait souligné avec clarté trois situations qui devaient être définies dans le cas particulier de la vie prénatale et de l'enfance: l'action thérapeutique simple (ou l'abstention thérapeutique) avec la notion de consentement éclairé du mineur, l'essai thérapeutique, enfin la recherche scientifique sans bénéfice individuel direct. Il me semble donc juste de reprendre quelques-uns de ses propos dont la pertinence n'a pas été mise en doute:

«Il est établi ainsi que beaucoup de problèmes de morale, d'éthique et de jurisprudence sont des problèmes relationnels. La personnalité de chacun y est confrontée au discours collectif. L'évolution de nos sociétés démontre que, quels que soient les désordres, les technologies définissent les morales et les règlements de l'avenir. Les symboles de la vieille société n'ont résisté ni au moteur à explosion, ni à la chaîne du froid, ni à la pilule de Pincus. Sans cesse, ainsi, avec des décalages, des tiraillements, des retours en arrière, des acceptations inattendues, la médecine de l'enfant dans ses actions de soins, d'essais thérapeutiques et de recherche finira par adapter ses symboles culturels à ses nouvelles technologies. "

Tant de responsabilités n'ont jamais écarté Pierre Royer de l'hôpital et de la recherche. Nombreux furent ceux qui trouvèrent auprès de lui le conseil décisif, le réconfort et l'aide qu'ils espéraient devant les difficultés des temps, les intolérances de toutes sortes et les exils cruels. Son rayonnement était presque fascinant et son écoute était toujours attentive et critique. Il savait avoir cette disponibilité étonnante, parfois impatiente, qui permettait l'échange de vue si précieux. La pédiatrie et la recherche biomédicale françaises retiendront Pierre Royer comme un des grands noms de progrès et d'humanisme dans cette deuxième moitié du siècle

\section{R. Rappaport}

Professeur des universités, hôpital des Enfants-Malades, 149, rue de Sèrres, 75015 Paris, France. 